\title{
An Evaluation of Burn Infections Caused by Multidrug Resistant A.baumannii and the Treatment Approach
}

\author{
Caferi Tayyar Selcuk ${ }^{\mathrm{a}, \mathrm{d}}$, Mehmet Bozkurt ${ }^{\mathrm{a}}$, Samet Vasfi Kuvat \\ Alicem Tekin ${ }^{\mathrm{b}}$, Recep Tekin ${ }^{\mathrm{c}}$, Emin Kapi ${ }^{\mathrm{a}}$
}

\begin{abstract}
Background: If not treated properly, A.baumannii infections in burn patients may cause delays in wound healing, graft losses, or even development of sepsis that may lead to death in cases where the bacteria enters the systemic circulation. In this study, our aim is to present the treatment options and their results in patients where burn infections due to multidrug resistant (MDR) A.baumannii have been diagnosed.
\end{abstract}

Methods: For the purpose of the study, 27 burn patients treated in our burn centre between June 2008 and January 2011, whose wound cultures revealed A.baumannii growth, were evaluated retrospectively. 16 among these patients $(59.2 \%)$ were male, whereas $11(40.8 \%)$ were female. The mean age of the patients was $32(1$ - 64) years and the total body surface area (TBSA) burned was 10 $65 \%(33.2 \%)$. The patients were evaluated in terms of compliance to the infection control and prevention standards, local wound treatment, systemic antibiotherapy according to the results of the antimicrobial sensitivity test, early surgical debridement and grafting.

Results: According to the results of the antimicrobial sensitivity test, the acinetobacteria were only sensitive to colistin. Full response to the treatment was achieved in all the patients where there was growth in the tissue cultures although no growth in the blood cultures was observed. Among the 5 patients whose blood cultures showed bacterial growth, 2 responded fully to the treatment where-

Manuscript accepted for publication November 9, 2011

${ }^{a}$ Department of Plastic Surgery, Dicle University, Faculty of Medicine, Diyarbakir, Turkey

${ }^{b}$ Department of Clinical Microbiology, Dicle University, Faculty of Medicine, Diyarbakir, Turkey

${ }^{c}$ Department of Infectious Disease and Clinical Microbiology, Dicle University, Faculty of Medicine, Diyarbakir, Turkey

${ }^{\mathrm{d}}$ Corresponding author: Caferi Tayyar Selcuk, Department of Plastic Surgery, Dicle University, Medical School, Yenisehir 21280 Diyarbakır, Turkey. Email: tayyarselcuk@hotmail.com

doi: $10.4021 / j \operatorname{cs} 105 \mathrm{e}$ as the other 3 patients (11.1\%) died of sepsis. The graft donor areas, grafts, and the superficial burn areas in all the patients healed without problem at the end of the treatment.

Conclusions: In the light of the information we obtained from our study, we believe that an early diagnosis of the wound infection, administration of the appropriate topical and systemic antimicrobial treatments, early surgical debridement and grafting is effective in controlling the infection in the management of the burn wounds infected with MDR A.baumannii.

Keywords: A.baumannii; Burn; Wound infection

\section{Introduction}

Although much progress has been made in the treatment of burn wounds, infection still continues to be the prominent cause of morbidity and mortality in burn patients [1-4]. The burn wounds carry a greater risk for bacterial contamination due to the damage on the skin which is the first barrier in the defence against infections, the avascular scar tissue providing a suitable environment for the growth of microorganisms and the suppression of the cellular immune system $[2,5,6]$. These conditions prepare the ground for the development of multidrug resistant (MDR) burn wound infections and sepsis.

A.baumannii, a gram-negative coccobacillus, seems to play a greater role in the hospital acquired infections in recent years [7]. Though this pathogen usually causes hospital acquired pneumonia cases, it has recently been reported as the etiological agent causing complicated infections such as wound infections, bacteraemia and osteomyelitis among burn patients and soldiers wounded in Afghanistan and Iraq [3, 7-14]. If not treated properly, it may cause delays in wound healing, graft losses, or development of sepsis and even death in cases where the bacteria enters the systemic circulation $[3,7,8]$. It is therefore important to take preventive measures before the infection develops. In order to do this, preventive treatment measures, the administration of the appropriate topical antibiotherapy, an early diagnosis of 
the wound infection together with an early excision of the infected burn scar tissue are required besides the continuous and regular infection control measures and the adoption of preventive standards [5, 14, 15].

In this study, we present the treatment modality applied in our burn centre and the results obtained in patients where burn wound infections caused by multidrug resistant (MDR) A.baumannii - a potential source for the development of sepsis - have been diagnosed.

\section{Material and methods}

Twenty-seven burn patients treated at the Dicle University Hospital Burn Centre between June 2008 and January 2011, in whose wound wipe samples and tissue biopsy cultures A.baumannii was isolated and identified as the infectious agent, have been prospectively enrolled in the study. Sixteen $(59.2 \%)$ out of the 27 patients enrolled were male whereas $11(40.8 \%)$ were female. The mean age of the patients was 32 ( 1 - 64) years. The total body surface area (TBSA) burned was determined as 10 - 65\% (mean: 33.2\%) (Table 1). Cases with alterations in the appearance of the burn wound (early scar separation; dark brown, black or purplish colouring in the scar; edema formation on the edges of the wound, etc.), a microorganism growth of $10^{5} \mathrm{cfu} / \mathrm{g}$ in the tissue biopsy cultures, or microorganism invasion to the live tissue detected during the histopathological assessment of the tissue biopsy sample obtained from the burn wound have been classified as burn wound infections [16]. Cases without these alterations have been evaluated as contaminations and exempted from the study. Also, in patients with more than a single growth, only the first growth has been included within the scope of the study.

The treatment applied, the interventions performed and the topical wound dressing agents used in each of the cases that have been classified as burn wound infections were recorded. The burn sites where A.baumannii has initially been diagnosed were accepted as the source of the infection.

\section{Microbiological methods}

The burn wound wipe cultures were obtained beginning from the first day when the patients were admitted to the burn centre and continued to be done routinely twice a week. Also tissue biopsy cultures were obtained from the patients whose burn wound wipe cultures revealed microorganism growth and whose burn wounds were altered in appearance. In patients under the suspicion of sepsis, also blood cultures were done. For these blood cultures, blood samples drawn from the peripheral vein were incubated in a BACTEC 9120 (Becton-Dickinson Diagnostic Inc., USA) device. The wipe and biopsy samples and the positive blood samples sent to the microbiology laboratory were inoculated into the surface

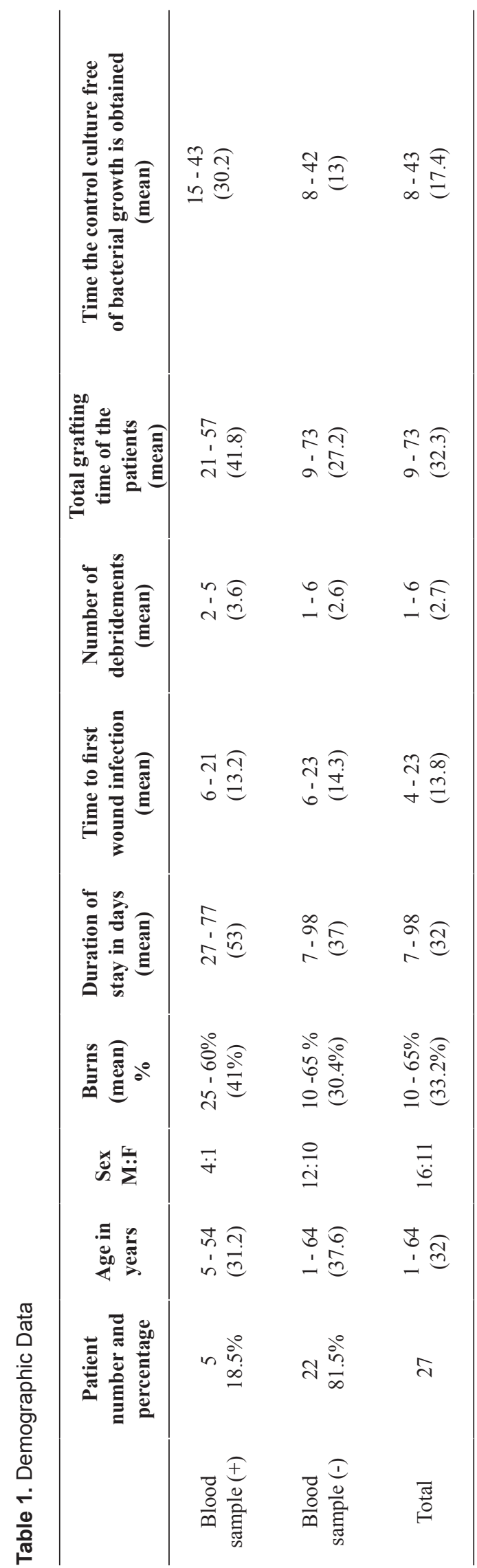


of an agar medium containing 5\% sheep blood and an EosinMethylene Blue agar medium. The colonies grown after the incubation were examined for identification. The amount of the colonies obtained from the tissue biopsy cultures were calculated as colony forming units per 1 gram of the tissue biopsy sample (cfu/g). The isolated colonies were identified both through the conventional methods (colony morphology, gram staining, oxidase testing, IMVIC characters), and using the Phoenix TM 100 (Becton Dickinson, USA) automated identification system. The antimicrobial sensitivity tests of the identified strains were performed through the Kirby-Bauer disk diffusion method in line with the recommendations of the Clinical and Laboratory Standards Institute (CLSI). The sensitivity to colistin has been studied using the Phoenix TM 100 (Becton Dickinson, USA) automated identification system and sensitivity has been established based on a value of $\mathrm{MIC} \leq 2.0 \mu \mathrm{g} / \mathrm{ml}$ according to CLSI guidelines.

\section{Assessment and management of burn patients}

Patients whose wipe sample and tissue biopsy cultures sent to the microbiology laboratory revealed A.baumannii growth were subjected to the standard isolation and infection control precautions. In line with the results of the antimicrobial sensitivity testing, the burn patients were started on a systemic colistin regimen of $4 \mathrm{mg} / \mathrm{kg} /$ day. While the superficial burn areas were managed through dressings only, a product with nanocrystallised silver content (Acticoat ${ }^{\mathrm{TM}}$, Smith and Nephew, Istanbul, Turkey), deep second degree and third degree burn areas have undergone early debridement and grafting. In patients with large burns where graft donor area was scarce, the debridements have been performed at intervals in order to protect the hemodynamic stability. In eight patients where the TBSA was $45 \%$ or more, cadaveric skin grafting has been applied subsequent to the debridement.

\section{Results}

The first growth of A.baumannii in the wound cultures of the patients, where a burn wound infection was diagnosed, was detected after an average of 13.8 days (between days 4 and 23). According to the results of the antimicrobial sensitivity testing, the acinetobacteria were only sensitive to colistin. While no growth was detected in the BAL, urine and catheter tip samples obtained from the nine patients where a sepsis was suspected, their wound cultures revealed bacterial growth. On the other hand, MDR A. baumannii was detected in the blood cultures of five patients and the antimicrobial sensitivity test revealed that the bacteria were only sensitive to colistin. All the patients whose tissue cultures showed bacterial growth although their blood cultures were free of growth achieved full response to the treatment. Among the 5 patients whose blood cultures indicated bacterial growth,

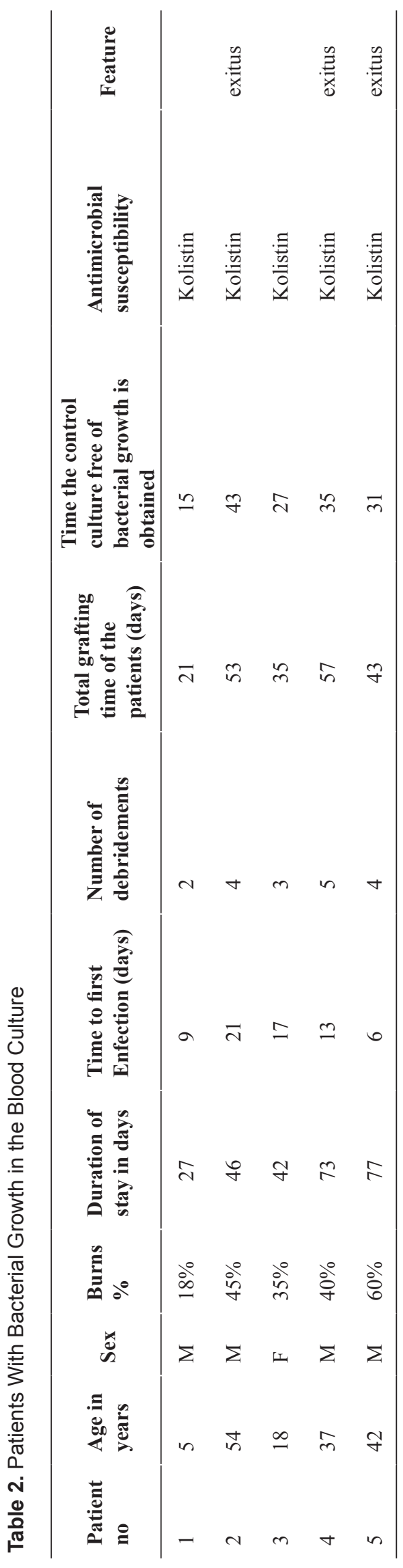


2 patients fully responded to the treatment while the other 3 $(11.1 \%$ ) died of sepsis (Table 2$)$. In our study, no bacterial growth has been detected in any of the wound and blood samples obtained from the patients after the treatment with colistin and the debridement. The graft donor areas, grafts and the superficial burn areas in all the patients healed without problem at the end of the treatment (Table 1).

\section{Discussion}

Although much progress has been made in the treatment of burn wounds, infection still continues to be a serious problem [3, 4]. A.baumannii can colonise in hospital burn units and may cause delays in wound healing, graft losses, sepsis and death $[3,7,8]$. Since A.baumannii has very efficient resistance development mechanisms, it is resistant to the great majority of the currently used antibiotics [7]. Therefore, starting the appropriate treatment before the agent leads to a systemic infection becomes important in burn patients where A.baumannii colonisation is detected. It has been shown in various studies that the adoption of infection control and prevention standards like proper hand hygiene, isolation rooms, environmental cleanliness and disinfection methods in burn units reduces infections caused by MDR microorganisms [17, 18]. Also in our case, these infection control and prevention standards were applied and the transmission of the infection to other patients was limited.

However, the leading step among the infection prevention measures is the local wound treatment. The topical antimicrobial products most commonly used in the local treatment of burn wounds are silver compounds [19-21]. Acticoat, a product with nanocrystallised silver content, has been reported in various studies to be safe in burn patients, to have greater microbicidal properties than classical silver compounds and to lead to a rapid healing in the wounds [2, 22-24]. In their experimental study, Uygur et al. [3] have demonstrated that Acticoat is effective in wound infections caused by MDR A.baumannii. Also, in another experimental study, Ulkur et al. [4] have shown that Acticoat limits MRSA development in the scar tissue and prevents its spread to the systemic circulation. Due to these properties, Acticoat has been used as the topical dressing product during our study and it has been observed to accelerate wound healing together with exerting a high antimicrobial activity. Kucan et al. [14] have demonstrated that mafenide acetate is more effective compared to silver sulfadiazine in A.baumannii-related wound infections. However, mafenide acetate has the disadvantage of causing metabolic acidosis when applied to larger burn areas $[15,25]$. There is an obvious need for further studies on this point.

Early surgical debridement and wound closure are other factors that play a role in the prevention of infections. The most important condition for the timely and effective appli- cation of these treatments is the daily assessment of the burn wounds by an experienced person. When a wound infection is diagnosed, the infected tissue must be removed immediately. In patients with larger burn areas, it is recommended to remove $10-20 \%$ of the burn area in a single debridement session and to continue the debridement at intervals of a few days $[15,17,25]$. In our study, when the wound was ready, grafting was performed immediately after the debridements. Nevertheless, in patients with larger burn areas, the need for repeated grafting and the waiting period for the regeneration of the donor areas prolong the process and increase the susceptibility to infection. In the eight patients in our study where there was a scarcity of graft donor area, in addition to the local wound management, cadaveric skin grafts have been applied following the debridement in order to protect the hemodynamic stability and to limit the spread of infection.

Another very important factor in infection control is the appropriate systemic antibiotherapy. Ganapathy et al. [26] have reported a $58 \%$ survival rate after the administration of colistin in MDR gram-negative infections in their burn centre. Trottier et al. [27] have observed a $37-87 \%$ resistance to imipenem, toramycin, amikacin and minocycline in patients with A.baumannii infections in their burn centre where all the cases were sensitive to colistin. They have reported the mortality rate following the treatment as $30 \%$. In our study, in all the patients where bacterial growth was detected in the tissue and blood samples, the antimicrobial sensitivity test results pointed out that A.baumannii was singly sensitive to colistin. Full response to the treatment was achieved in all the patients where there was bacterial growth in the tissue cultures although no growth in the blood cultures was observed. Among the 5 patients whose blood cultures revealed bacterial growth, 2 responded fully to the treatment whereas the other 3 patients $(11.1 \%)$ died of sepsis.

In various studies, the mortality rate due to MDR A.baumannii infections among burn patients has been reported as $30-38 \%[13,27-29]$. The $11 \%$ mortality in our study was found to be lower in comparison to these mortality rates reported in the literature. We believe that this lower mortality rate is due to both the inclusion of only burn wound infections into our study and to an attitude combining an early diagnosis of wound infections with the appropriate topical and systemic antimicrobial treatments, surgical debridements and early wound closure.

In the light of the data obtained from our study, we are of the opinion that a combination of an early diagnosis of wound infections, appropriate topical and systemic antimicrobial treatments, surgical debridement and early wound closure may be effective in the management of burn wounds infected with MDR A.baumannii. Also, the adoption of infection control and prevention standards like proper hand hygiene, isolation rooms, environmental cleanliness and disinfection methods may significantly reduce infections caused 
by MDR microorganisms in burn units.

\section{References}

1. Zhang XJ, Heggers JP, Chinkes DL, Wolf SE, Hawkins HK, Wolfe RR. Topical Sulfamylon cream inhibits DNA and protein synthesis in the skin donor site wound. Surgery. 2006;139(5):633-639.

2. Fong J, Wood F. Nanocrystalline silver dressings in wound management: a review. Int $\mathrm{J}$ Nanomedicine. 2006;1(4):441-449.

3. Uygur F, Oncul O, Evinc R, Diktas H, Acar A, Ulkur E. Effects of three different topical antibacterial dressings on Acinetobacter baumannii-contaminated full-thickness burns in rats. Burns. 2009;35(2):270-273.

4. Ulkur E, Oncul O, Karagoz H, Yeniz E, Celikoz B. Comparison of silver-coated dressing (Acticoat), chlorhexidine acetate $0.5 \%$ (Bactigrass), and fusidic acid $2 \%$ (Fucidin) for topical antibacterial effect in methicillinresistant Staphylococci-contaminated, full-skin thickness rat burn wounds. Burns. 2005;31(7):874-877.

5. Uygur F, Özyurt M, Evinç R, Hosbul T, Çeliköz B, Haznedaroglu T. Comparison of octenidine dihydrochloride (Octenisept ${ }^{\circledR}$ ), polihexanide (Prontosan ${ }^{\circledR}$ ) and povidon iodine (Betadine $\left.{ }^{\circledR}\right)$ for topical antibacterial effects in Pseudomonas aeruginosa-contaminated, full-skin thickness burn wounds in rats. Cent Eur J Med. 2008;3:417-421.

6. Gravante G, Caruso R, Sorge R, Nicoli F, Gentile P, Cervelli V. Nanocrystalline silver: a systematic review of randomized trials conducted on burned patients and an evidence-based assessment of potential advantages over older silver formulations. Ann Plast Surg. 2009;63(2):201-205.

7. Mihu MR, Sandkovsky U, Han G, Friedman JM, Nosanchuk JD, Martinez LR. The use of nitric oxide releasing nanoparticles as a treatment against Acinetobacter baumannii in wound infections. Virulence. 2010;1(2):62-67.

8. Davis SC, Pisanni F, Montero RB. Effects of commonly used topical antimicrobial agents on Acinetobacter baumannii: an in vitro study. Mil Med. 2008;173(1):74-78.

9. Maragakis LL, Perl TM. Acinetobacter baumannii: epidemiology, antimicrobial resistance, and treatment options. Clin Infect Dis. 2008;46(8):1254-1263.

10. Keen EF, 3rd, Robinson BJ, Hospenthal DR, Aldous WK, Wolf SE, Chung KK, Murray CK. Prevalence of multidrug-resistant organisms recovered at a military burn center. Burns. 2010;36(6):819-825.

11. Ganapathy H, Pal SK, Teare L, Dziewulski P. Use of colistin in treating multi-resistant Gram-negative organisms in a specialised burns unit. Burns. 2010;36(4):522-527.

12. Chim H, Tan BH, Song C. Five-year review of infections in a burn intensive care unit: High incidence of
Acinetobacter baumannii in a tropical climate. Burns. 2007;33(8):1008-1014.

13. Trottier V, Segura PG, Namias N, King D, Pizano LR, Schulman CI. Outcomes of Acinetobacter baumannii infection in critically ill burned patients. J Burn Care Res. 2007;28(2):248-254.

14. Kucan JO, Heggers JP. The potential benefit of 5\% Sulfamylon Solution in the treatment of Acinetobacter baumannii-contaminated traumatic war wounds. J Burns Wounds. 2005;4:e3.

15. Young DM. Burn and electrical injury. Plastic Surgey, second edition, edited by Mates SJ, Sounders Elsevier Publishers, Philadelphia, volume 1, p: 811-833, 2006.

16. Mayhall CG. Nosocomial burn wounds. In Mayhall CG (ed.): Hospital Epidemiology and Infection Control. Second edition, Lippincot Williams \& Wilkins, Philadelphia, p: 275- 86, 1999.

17. D'Avignon LC, Saffle JR, Chung KK, Cancio LC. Prevention and management of infections associated with burns in the combat casualty. J Trauma. 2008;64(3 Suppl):S277-286.

18. Hemmila MR, Mattar A, Taddonio MA, Arbabi S, Hamouda T, Ward PA, Wang SC, et al. Topical nanoemulsion therapy reduces bacterial wound infection and inflammation after burn injury. Surgery. 2010;148(3):499-509.

19. Bragg PD, Rainnie DJ. The effect of silver ions on the respiratory chain of Escherichia coli. Can J Microbiol. 1974;20(6):883-889.

20. Tshukudu GM, van der Walt M, Wessels Q. Comparative in vitro study of honey based and silver based wound preparations on cell viability. Burns. 2010;36(7):10361041.

21. Moiemen NS, Shale E, Drysdale KJ, Smith G, Wilson YT, Papini R. Acticoat dressings and major burns: systemic silver absorption. Burns. 2011;37(1):27-35.

22. Carter MJ, Tingley-Kelley K, Warriner RA, 3rd. Silver treatments and silver-impregnated dressings for the healing of leg wounds and ulcers: a systematic review and meta-analysis. J Am Acad Dermatol. 2010;63(4):668679.

23. Dunn K, Edwards-Jones V. The role of Acticoat with nanocrystalline silver in the management of burns. Burns. 2004;30 Suppl 1:S1-9.

24. Press B. Thermal, electrical and chemical injuries. Grabb and Smith's Plastic Surgey, Fifth edition, edited by SJ Aston, RW Beasley and CHM Thorne. LippincottRaven Publishers, Philadelphia, p:161-89, 1997.

25. Bang RL, Gang RK, Sanyal SC, Mokaddas E, Ebrahim MK. Burn septicaemia: an analysis of 79 patients. Burns. 1998;24(4):354-361.

26. Sengupta S, Kumar P, Ciraj AM, Shivananda PG. Acinetobacter baumannii--an emerging nosocomial pathogen in the burns unit Manipal, India. Burns. 2001;27(2):140-144. 